\title{
Selective Determination of Uric Acid in the Presence of Ascorbic Acid Using Layer-by-Layer Gold Nanoparticles, Tin Oxide Nanoparticles and Multi-walled Carbon Nanotubes Assembled Multilayer Films
}

\author{
Yan PAN, ${ }^{a}$ Yong LI, ${ }^{\mathbf{b}, *}$ Juanying JIA, $^{a}$ and Hongmei CHEN ${ }^{\mathrm{b}}$ \\ a Analysis and Testing central Facility of Anhui University of Technology, \\ Maanshan 243000, People's Republic of China \\ b School of Mathematics \& Physics, Anhui University of Technology, \\ Maanshan 243000, People's Republic of China \\ *Corresponding author: yongli@ahut.edu.cn
}

\begin{abstract}
Multi-walled carbon nanotubes (MWCNTs) and gold nanoparticles (Au NPs) were assembled on glassy carbon electrode (GCE) to form homogeneous and stable multilayer films using a layer-by-layer assembled method by electrostatic interaction. Electrochemical impedance spectroscopy (EIS), Cyclic voltammetry (CV), Scanning electron microscopy (SEM), Transmission electron microscopy (TEM), and Ultraviolet-visible absorption spectroscopy (UV) were used for characterization of multilayer films. Electrocatalytic activity of uric acid (UA) was studied. UA and ascorbic acid (AA) of electrochemical signals were well separated on the modified electrode in $\mathrm{pH} 7.0$ phosphate buffered solution (PBS). The oxidation peak potential of AA and UA was appeared at -91 and $247 \mathrm{mV}$ vs. SCE on differential pulse voltammograms (DPVs), respectively. Response of UA was linear in the range from $4.0 \times 10^{-6} \mathrm{~mol} / \mathrm{L}$ to $3.0 \times 10^{-3} \mathrm{~mol} / \mathrm{L}$ and linear regression equation was $\mathrm{i}_{\mathrm{pa}}(\mu \mathrm{A})=1.027+0.037 \mathrm{C}(\mu \mathrm{mol} / \mathrm{L})$ with a correlation coefficient of 0.992 , the limit of detection for UA was $2.0 \times 10^{-6} \mathrm{~mol} / \mathrm{L}$.
\end{abstract}

(C) The Electrochemical Society of Japan, All rights reserved.

Keywords : Gold Nanoparticles, Multi-walled Carbon Nanotubes, Tin Oxide Nanoparticles, Uric Acid

\section{Introduction}

Due to their abundant electronic, chemical and mechanical properties, Carbon nanotubes (CNTs) are widely applied. ${ }^{1,2}$ However, their poor solubility and biological incompatibility impose restrictions on application. ${ }^{3-6}$ Therefore, it is important to synthesis CNTs with different kinds of morphologies and properties, and this can enlarge their applications in various fields including composites, electrochemical devices and sensors. ${ }^{7-9}$ At present, CNTs are hindered to be applied in electrochemical sensors due to nonuniform film coverage, lack control of film properties and difficulty in preparing complex film architectures with several different components. ${ }^{10}$

Due to their good biocompatibility, enhanced electrocatalytic activity, large surface area and excellent conductivity, Gold nanoparticles ( $\mathrm{Au} \mathrm{NPs}$ ) have been used in development of electrochemical sensors and biosensors on their catalytic activities. ${ }^{11,12}$ Nano$\mathrm{SnO}_{2}$ have advantages of excellent optical and electrical properties, high sensitivities of gas and short response time, as well as relatively higher conductivity than $\mathrm{TiO}_{2}$ and $\mathrm{SiO}_{2} \cdot{ }^{13-15} \mathrm{New}$ types of inorganic nanoparticle materials exhibit adjustable porosity and high thermal and chemical stability. As natural existence of deacetylation of chitin, chitosan (CHIT) is a polysaccharide extracted from crustaceans, and it exhibits a positive charge in acidic solution. In addition, it has properties of biodegradability and biocompatibility. ${ }^{16,17}$ Its degradation products, which show no toxicity and immunogenicity, do not accumulate in the body. Good membrane and biological safety of chitosan make it have a wide application in the development of sensors.

Based on electrostatic interaction, the layer-by-layer (LBL) selfassembly technique attracts comprehensive interest because of its simple process, a variety of available materials, precise control of composition and thickness of layer in the molecular level. ${ }^{18-20}$ This technique was originally developed by Decher and his coworkers $^{21,22}$ for linear polyelectrolyte. In recent years, the LBL self-assembly technique is applied to sensor devices. For example, Pauli et al assembled cashew gum/phaeophytin-b multilayer films by LBL self-assembling and investigated its application on $\mathrm{H}_{2} \mathrm{O}_{2}$ biosensors. ${ }^{23}$ Kang et al fabricated multilayer of PDA-MWCNT/ enzyme composite films for the electrochemical detection of nonesterified fatty acid. ${ }^{24}$

In order to make full use of the advantages of Au NPs, Nano$\mathrm{SnO}_{2}$, and CNTs, we designed the experiment. We prepared [CHIT$\mathrm{SnO}_{2} / \mathrm{Au}$ NPs/CHIT-SnO $/$ MWCNTs $\}_{n}$ multilayer films modified GCE using a layer-by-layer assembled method by electrostatic interaction, which showed good electrocatalytic activity towards uric acid oxidation. The modified electrode could be applied to detecting UA in the presence of AA. And modified electrode of the multilayer film assembled by LBL has advantages over other modified electrode in nanoparticles individually and any of two composite nanoparticles based on the result of experiment.

\section{Experiment Details}

\subsection{Reagents and apparatus}

Multi-walled carbon nanotubes with carboxylic acid groups were purchased from the Chengdu Institute of Organic Chemistry, Chinese Academy of Sciences and were used without further purification (purity $>90 \%$ ). Chitosan, UA, and AA were purchased from Sigma. Other chemicals were of analytical reagent grade. Phosphate buffered saline solutions (PBS; $0.1 \mathrm{~mol} / \mathrm{L}$ ) with different $\mathrm{pH}$ values were prepared by mixing the stock standard solutions with $\mathrm{K}_{2} \mathrm{HPO}_{4}$ and $\mathrm{KH}_{2} \mathrm{PO}_{4}$, and the $\mathrm{pH}$ value was adjusted with $\mathrm{H}_{3} \mathrm{PO}_{4}$ or $\mathrm{NaOH}$. All solutions were prepared with double-distilled water.

CVs and DPVs were obtained on a CHI650C electrochemical workstation (Shanghai Chenhua Instrument Company, China) with a 


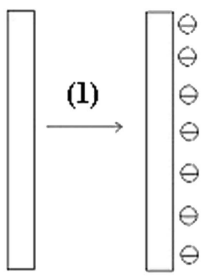

GCE

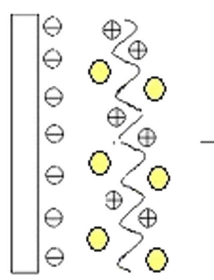

(3)

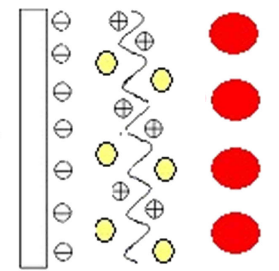

(4)

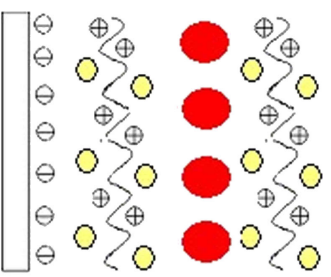

$\mathrm{CHTT}-\mathrm{SnO}_{2}$

Gold nanoparticles

(5)

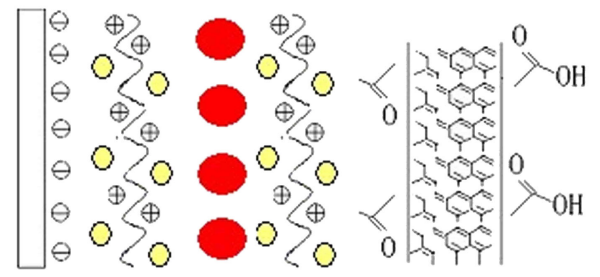

Oxide MWCNTs

Scheme 1. (Color online) Assembly process of the $\left\{\mathrm{CHIT}-\mathrm{SnO}_{2} / \mathrm{GNPs} / \mathrm{CHIT}-\mathrm{SnO}_{2} / \mathrm{MWCNTs}\right\}_{\mathrm{n}}$ multilayer films.

three-electrode system. A platinum wire was used as an auxiliary electrode, saturated calomel electrode (SCE) as a reference electrode and a bare and multi-layer films modified electrode as a working electrode.

Scanning electron microscopy (SEM) images were obtained with a JSM-6700F scanning electron, Transmission electron microscopy (TEM) images with a JEM-2100F, UV-vis absorption spectroscopy with a SHIMADZU-2450 and charged data with a ZETA potential instrument (Malvern Zetasizer nano 90).

\subsection{Preparation of $\mathrm{SnO}_{2} \mathrm{NPs}$}

$\mathrm{SnO}_{2} \mathrm{NPs}$ was prepared by hydrothermal synthesis. Appropriate amounts of $0.8790 \mathrm{~g} \mathrm{SnCl}_{4} \cdot 5 \mathrm{H}_{2} \mathrm{O}$ were dissolved in a mixture of $10 \mathrm{ml}$ glycol and $50 \mathrm{ml}$ water, then it was used to adjust $\mathrm{pH}$ of the solution to 1 and reacted $24 \mathrm{~h}$ at $150^{\circ} \mathrm{C}$. The products were washed with ethanol and dried at $80^{\circ} \mathrm{C}$.

\subsection{Preparation of Au NPs}

$\mathrm{Au}$ NPs was prepared according to this report. ${ }^{25}$ In a $500 \mathrm{ml}$ beaker, $4.0 \mathrm{ml} 1 \%$ sodium citrate was quickly added to $250 \mathrm{ml}$ $0.01 \%$ boiling $\mathrm{HAuCl}_{4}$ solution under vigorous stirring. Its color changed from yellow to red violet. The mixture was boiled continuously for $10 \mathrm{~min}$ under stirring to produce $\mathrm{Au}$ colloidal particles. Au NPs obtained were stored at $4^{\circ} \mathrm{C}$ in a dark bottle.

\subsection{Preparation of the multilayer film modified electrode}

Before surface modification, bare glassy carbon electrode (GCE) $(3.0 \mathrm{~mm}$ in diameter) was freshly polished with $1.0,0.3$, and $0.05 \mu \mathrm{m}$ alumina powder, respectively. Then the electrode was cleaned ultrasonically sequentially in ethanol and water for $3.0 \mathrm{~min}$. Finally, the electrode was electrochemically cleaned between $-0.3 \mathrm{~V}$ and $+1.50 \mathrm{~V}$ by repetitive cyclic potential scan for 20 cycles in $0.50 \mathrm{~mol} / \mathrm{L}$ sulfuric acid to remove any possible impurities on the surface. The glassy carbon electrode was activated to increase oxygen group for $-\mathrm{OH},-\mathrm{COOH},-\mathrm{C}=\mathrm{O}$ on the electrode surface. Existence of many oxygen groups is generated on the electrode surface with a negative charge. ${ }^{26-28}$

$10.0 \mathrm{mg}$ MWCNTs was dispersed in $10.0 \mathrm{~mL}$ water with the aid of ultrasonic agitation to produce $1.0 \mathrm{mg} \mathrm{mL}^{-1}$ suspension. $0.25 \mathrm{wt} \%$ chitosan was obtained with suitable chitosan dissolved in $0.1 \mathrm{~mol} / \mathrm{L} \mathrm{HCl}$ solutions. $\mathrm{SnO}_{2} \mathrm{NPs}$ were dispersed into aqueous solution of $0.25 \mathrm{wt} \%$ chitosan by ultrasonic agitation $12 \mathrm{~h}$ to give a homogeneous suspension.
$\left\{\mathrm{CHIT}_{-} \mathrm{SnO}_{2} / \mathrm{Au} \mathrm{NPs} / \mathrm{CHIT}-\mathrm{SnO}_{2} / \mathrm{MWCNTs}_{\mathrm{n}}\right.$ multilayer films were obtained, and Schematic diagram showing the general procedure is presented in Scheme 1. First, the pretreated GC electrode was immersed into positively charged CHIT- $\mathrm{SnO}_{2}$ (zeta potential is $29.2 \mathrm{mv}$ ) and negatively charged Au NPs (zeta potential is $-45.8 \mathrm{mv}$ ) in sequence, then positively charged CHIT-SnO 2 was immersed once again. Finally MWCNTs was immersed on the external surface to form a layer of $\{$ CHIT-SnO $2 / \mathrm{Au}$ NPs/CHIT-SnO $2 /$ MWCNTs $\}$. Assembling time for each layer was $30 \mathrm{~min}$., and the electrode was well washed with double distilled water after each assembly.

\section{Result and Discussion}

\subsection{TEM images of $\mathrm{Au}$ NPs and $\mathrm{SnO}_{2} \mathrm{NPs}$}

Figure $1 \mathrm{~A}$ and $1 \mathrm{~B}$ show the typical TEM image and UV spectroscopy of Au NPs. Particles were homogeneous with average diameter of $13 \mathrm{~nm}$ from TEM (Fig. 1A), which is in accordance with a $13 \mathrm{~nm} \mathrm{Au} \mathrm{NPs} \mathrm{of} \mathrm{UV} \mathrm{absorption} \mathrm{peak} \mathrm{at} 520 \mathrm{~nm}$ (Fig. 1B). $\mathrm{SnO}_{2}$ NPs were homogeneous with average diameter of $20-25 \mathrm{~nm}$ from TEM by hydrothermal synthesis (Fig. 1C).

\subsection{SEM images of the multilayer films}

Assembly and morphology of $\{$ CHIT-SnO $2 / \mathrm{Au}$ NPs/CHIT$\mathrm{SnO}_{2} /$ MWCNTs $\}_{\mathrm{n}}$ multilayer films were characterized by SEM. Figure 2 displays typical SEM images of the outermost layer of the multilayer films. The films consist of 1, 2 and 3 outermost layers of MWCNTs (panels A, B and C, respectively) assembled on the glass carbon electrode (the assembled processes were according to Scheme 1). As a result, only nanotubes could be seen in the images due to invisibility of inner layers of nano- $\mathrm{SnO}_{2}$ and Au nanoparticles. The first layer, a composite film composed of tangled MWCNTs, shows a uniform morphology (Fig. 2A). Subsequently, the second layer, the degree of coverage of MWCNTs is much higher than that of the first layer and relatively separated MWCNTs are interlinked partially (Fig. 2B). For the third layer (Fig. 2C). The surface is almost covered completely with MWCNTs. Gradual growth of the films indicates the successful electrostatic LBL assembly.

\subsection{UV absorption spectroscopy}

In order to measure absorption spectra, multilayer films was assembled on quartz slides. Figure 3 shows absorbance spectra of multilayer films from one to eight layers. The absorbance peak of $\mathrm{Au}$ NPs locates at $540 \mathrm{~nm}$ and MWCNTs locate at $268 \mathrm{~nm}$ for the 
(A)
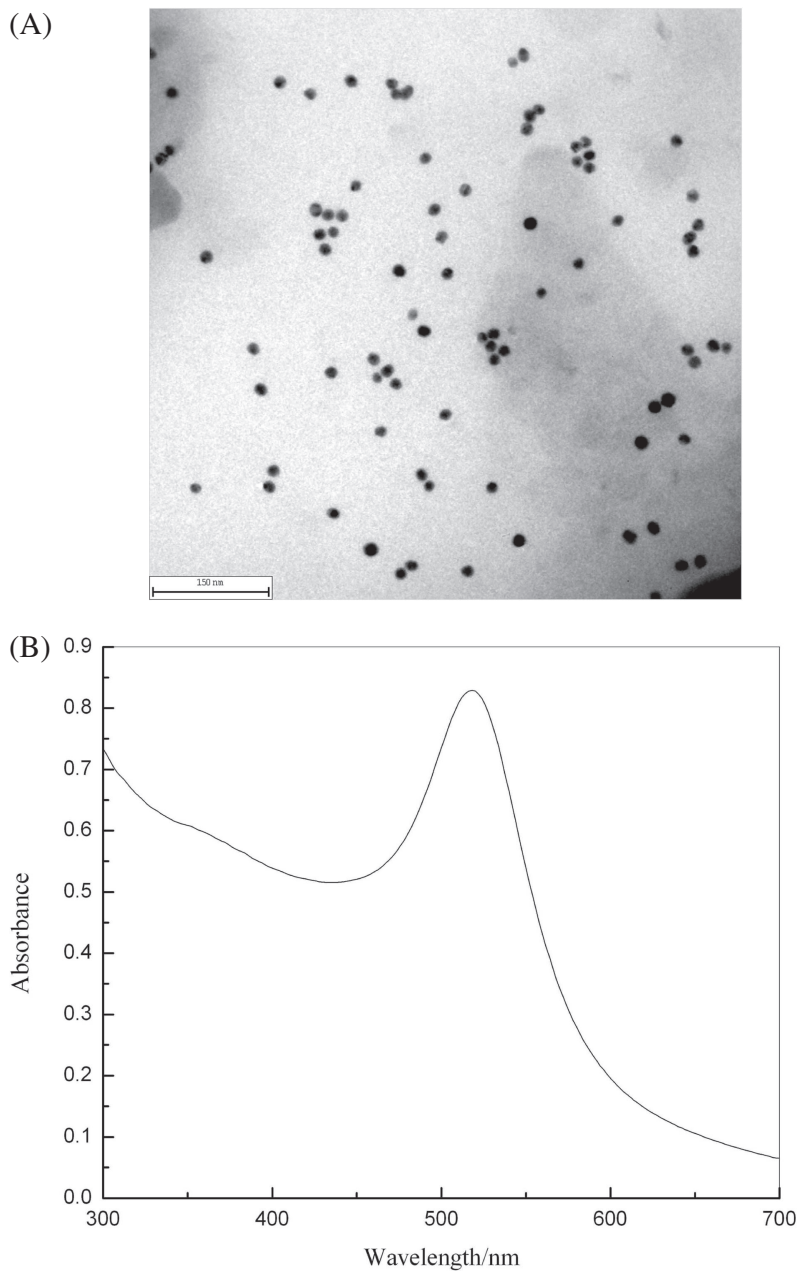

(C)

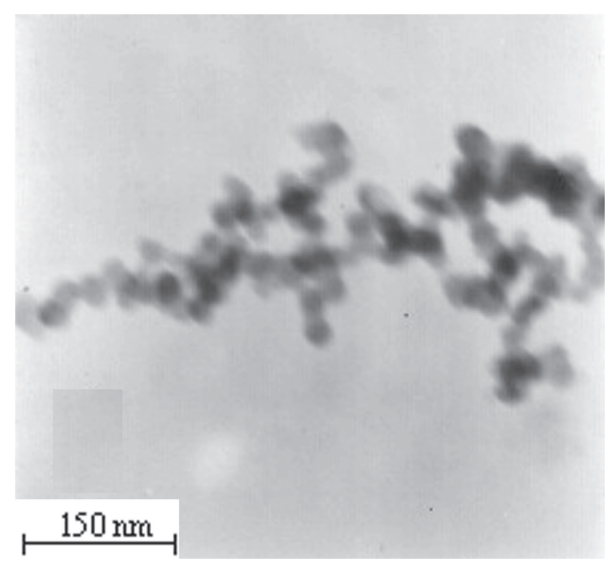

Figure 1. (A) TEM image of Au NPs; (B) UV-Vis spectroscopy of $\mathrm{Au}$ NPs; (C) TEM image of $\mathrm{SnO}_{2}$ NPs.

first layer film, respectively. Absorption peaks of $\mathrm{Au}$ NPs and MWCNTs become broader and shift respectively to $600 \mathrm{~nm}$ and $290 \mathrm{~nm}$ due to irreversible aggregation of Au NPs and MWCNTs when the layer number is increased (Fig. 3A). Absorbance enhances with layers number increased and has a linear relationship with the number of layer increased from one to eight layers (Fig. 3B). This indicates that a progressive and uniform deposition process of multilayer films and same amount of adsorbed Au NPs and MWCNTs for each layer during the assembly process.

\subsection{Cyclic voltammograms of the GCE modified electrode}

Figure 4 shows $\mathrm{CVs}$ of the $5.0 \times 10^{-3} \mathrm{~mol} / \mathrm{L}\left[\mathrm{Fe}(\mathrm{CN})_{6}\right]^{4-/ 3-}$ at the different film modified electrodes in $0.1 \mathrm{~mol} / \mathrm{L} \mathrm{KCl}$ solution. As
(A)

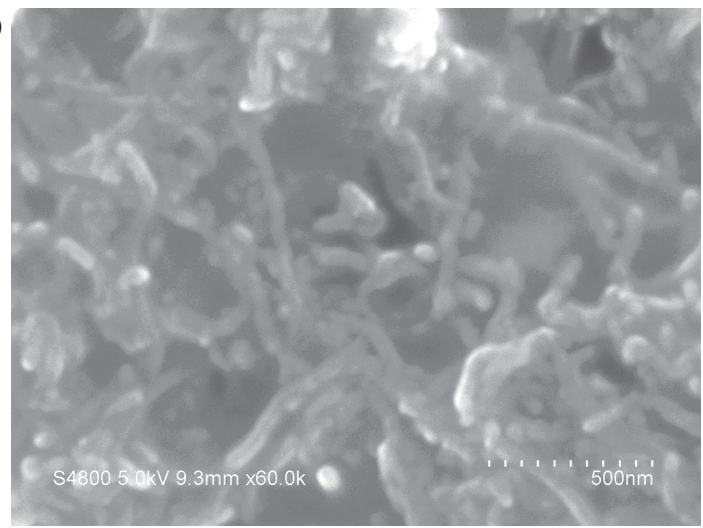

(B)

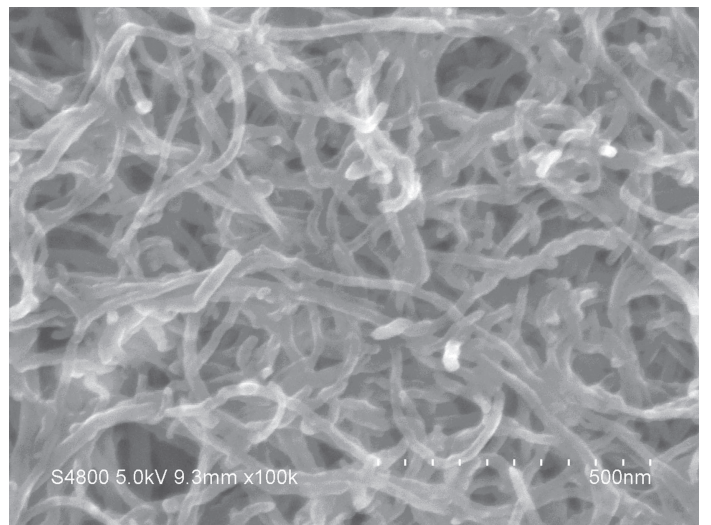

(C)

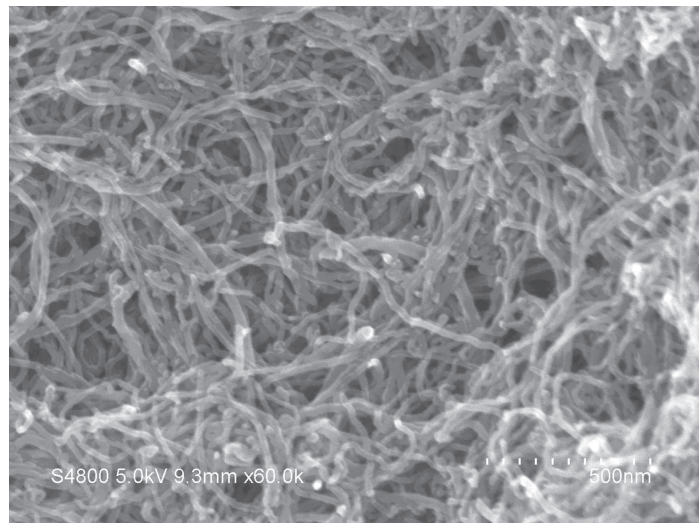

Figure 2. SEM images of $\left\{\mathrm{CHIT}-\mathrm{SnO}_{2} / \mathrm{Au}\right.$ NPs/CHIT-SnO ${ }_{2} /$ MWCNTs $\}_{\mathrm{n}}$ multilayer films (A) $\mathrm{n}=1$; (B) $\mathrm{n}=2$; (C) $\mathrm{n}=3$.

shown in Fig. 4A, a couple of reversible and weak redox peaks of probes are observed in the bare GC electrode (curve b). When the GC electrode is assembled with chitosan, response of the probe (curve a) becomes weaker than that on the bare electrode (curve b). This is due to poor conductivity of chitosan. The CHIT-SnO 2 modified electrode (curve c) shows higher electrochemical response than chitosan modified electrode (curve b) because $\mathrm{SnO}_{2} \mathrm{NPs}$ have good electrical properties and a lager surface area. ${ }^{29,30}$ When $\mathrm{Au}$ NPs are introduced to the surface (curve d), the anodic peak current is higher than that at the CHIT-SnO ${ }_{2}$ modified electrode. This phenomenon can be ascribed to the excellent electron transfer ability of Au NPs. When MWCNTs is introduced to the surface of GCE (curve e, f, g), the peak current is significantly increased because of the excellent electron transfer ability of MWCNTs which improved electron transfer and mass transfer of $\left[\mathrm{Fe}(\mathrm{CN})_{6}\right]^{4-/ 3-}$ ions on electrode interface. ${ }^{31,32}$ The assembled composite electrode interface based on the $\mathrm{SnO}_{2}$ NPs, Au NPs and MWCNTs improves electrode electrochemical performances. Performances of electrodes modified 

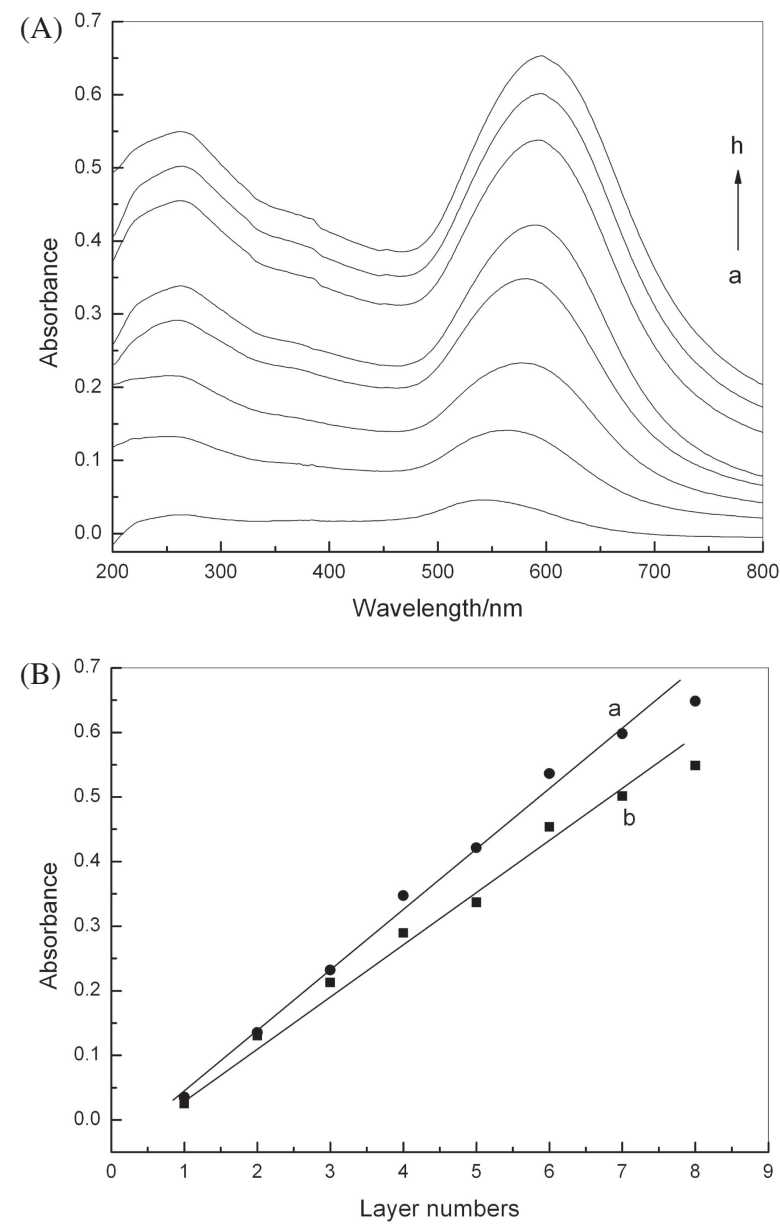

Figure 3. (A) UV-vis absorption spectra of $\left\{\mathrm{CHIT}-\mathrm{SnO}_{2} / \mathrm{Au}\right.$ $\left.\mathrm{NPs} / \mathrm{CHIT}-\mathrm{SnO}_{2} / \mathrm{MWCNTs}\right\}_{\mathrm{n}}$ multilayer films on the quartz slides with different layer number: $\mathrm{n}=1,2,3,4,5,6,7,8$; (B) The relationship between the number of layers and the absorbance value of (a) Au NPs (b) MWCNTs.

with only MWCNTs (curve e) and CHIT-SnO 2 (curve c) were also evaluated, it is shown that the peak current becomes higher than that of the bare GC electrode (curve a). However, the peak current of only MWCNTs (curve e) modified electrode is smaller than that of CHIT-SnO 2 /MWCNTs/GCE (curve f) and Au NPs/MWCNTs/ GCE (curve g). Similarly, the peak current of only CHIT-SnO (curve c) modified electrode was smaller than that of CHIT- $\mathrm{SnO}_{2} /$ MWCNTs/GCE (curve f) and Au NPs/CHIT-SnO $/$ GCE (curve d). This result demonstrates that the assembled composite electrode has superior electrochemical performances to those modified with MWCNTs, or CHIT-SnO ${ }_{2}$ individually. When the electrode was modified using the layer-by-layer assembled method by electrostatic interaction, $\mathrm{Au}$ NPs, CHIT-SnO ${ }_{2}$ and MWCNTs were imported together on the electrode, the peak current (curve a of Fig. 4B) has much more significant increase than all the other modified electrode, and the peak current value is about 13 times than that of Au NPs/ MWCNTs modified electrodes. The result shows nanocomposite electrodes not only have the basic performance of individual nanoparticles, which can improve the electron transfer, but also composite synergistic multifunctional effect further improving electrochemical performances. As shown in Fig. 4B, the oxidation peak current increases obviously after assembling the multilayer films of $\left\{\right.$ CHIT-SnO $2 / \mathrm{Au}$ NPs $/ \mathrm{CHIT}-\mathrm{SnO}_{2} / \mathrm{MWCNTs}_{\mathrm{n}}$ onto the GCE surface. This can be attributed to the synergistic effect of MWCNTs, $\mathrm{SnO}_{2}$ and $\mathrm{Au}$ NPs, promoting the electron transfer between the redox probe and the electrode surface. ${ }^{33-35}$ Figure $4 \mathrm{C}$ shows CVs of the multi-layers film modified electrode in $\mathrm{pH} 7.0$
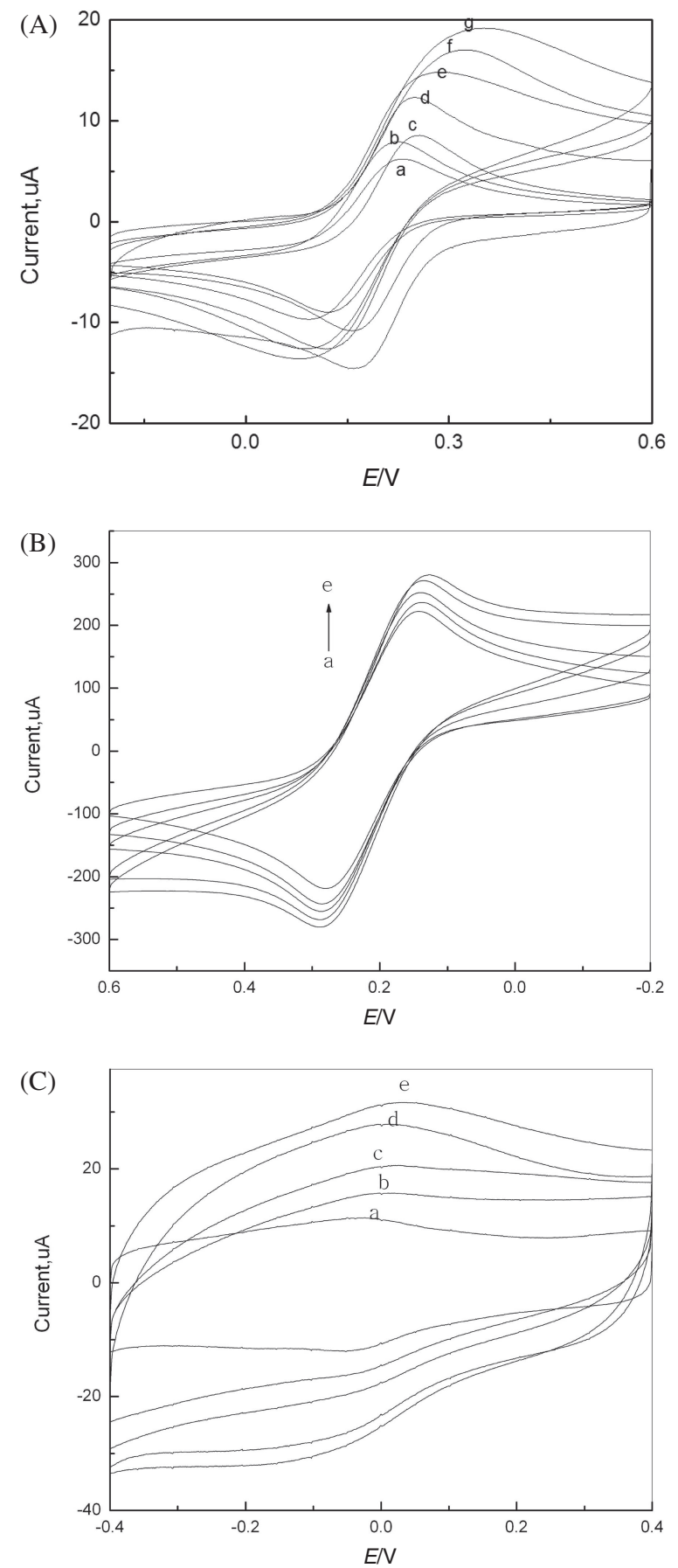

Figure 4. (A) Voltammetric responses of the glass carbon electrode at different stages to $5.0 \times 10^{-3} \mathrm{~mol} / \mathrm{L} \mathrm{K} \mathrm{K}_{3}\left[\mathrm{Fe}(\mathrm{CN})_{6}\right] /$ $\mathrm{K}_{4}\left[\mathrm{Fe}(\mathrm{CN})_{6}\right]$ and $0.1 \mathrm{~mol} / \mathrm{L} \mathrm{KCl}$ solution at $100 \mathrm{mV} \mathrm{s}^{-1}$, Panel a, b, c, d, e, f, g corresponding to (a) chitosan/GCE, (b) bare GCE, (c) CHIT-SnO $2 /$ GCE, (d) CHIT-SnO $2 /$ GNPs/GCE, (e) MWCNTs/ GCE, (f) CHIT-SnO $2 /$ MWCNTs/GCE, (g) Au NPs/MWCNTs/ GCE, respectively; (B) Voltammetric responses of the glass carbon electrode at different stages to $5.0 \times 10^{-3} \mathrm{~mol} / \mathrm{L} \mathrm{K} \mathrm{K}_{3}\left[\mathrm{Fe}(\mathrm{CN})_{6}\right] /$ $\mathrm{K}_{4}\left[\mathrm{Fe}(\mathrm{CN})_{6}\right]$ and $0.1 \mathrm{~mol} / \mathrm{L} \mathrm{KCl}$ solution at $100 \mathrm{mV} \mathrm{s}^{-1}$, Panel $\mathrm{a}, \mathrm{b}, \mathrm{c}, \mathrm{d}$, e corresponding to $\mathrm{n}=1,2,3,4,5$, respectively; (C) $\mathrm{CVs}$ corresponding to the $\left\{\mathrm{CHIT}-\mathrm{SnO}_{2} / \mathrm{Au} \mathrm{NPs} / \mathrm{CHIT}-\mathrm{SnO}_{2}\right.$ / MWCNTs $\}_{\mathrm{n}}$ multilayer films assembled at the glass carbon electrode in $\mathrm{pH}$ 7.0 PBS with $\mathrm{n}=1,2,3,4,5$, the scan rate is $100 \mathrm{mV} \mathrm{s}^{-1}$.

PBS from 1 to 5 . In the potential ranging from -0.4 to $0.4 \mathrm{~V}$, a pair of redox peaks near zero potential are observed. The redox peaks current increases with the growing layer number, it is confirmed that redox peaks are attributed to MWCNTs. It suggests that 


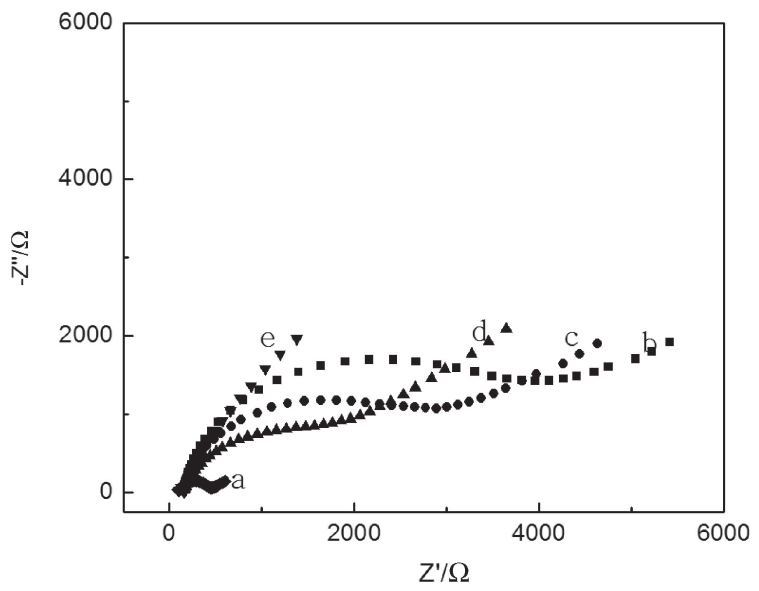

Figure 5. Nyquist diagram $\left(Z^{\prime \prime}\right.$ vs. $\left.Z^{\prime}\right)$ of the different modified $\mathrm{GC}$ electrodes in the presence of $5.0 \times 10^{-3} \mathrm{~mol} / \mathrm{L} \mathrm{K}_{3}\left[\mathrm{Fe}(\mathrm{CN})_{6}\right] /$ $\mathrm{K}_{4}\left[\mathrm{Fe}(\mathrm{CN})_{6}\right]$ and $0.1 \mathrm{~mol} / \mathrm{L} \mathrm{KCl}$ solution at electrode potential for $0.19 \mathrm{~V}$ (a) Bare electrode, (b) Chitosan/GCE, (c) CHIT-SnO $2 / \mathrm{GCE}$, (d) $\mathrm{CHIT}-\mathrm{SnO}_{2} / \mathrm{Au} \mathrm{NPs} / \mathrm{GCE}$, (e) CHIT-SnO $2 / \mathrm{Au}$ NPs/CHIT$\mathrm{SnO}_{2} / \mathrm{MWCNTs} / \mathrm{GCE}$.

electrochemical properties of MWCNTs are not changed in the multilayer films.

\subsection{Electrochemical impedance characterization of different modified electrodes}

Impedance spectroscopy is an effective method for probing surface features of the modified electrode. ${ }^{36}$ In EIS, the semicircle part at higher frequency corresponds to the limited process of electron transfer and the diameter of semicircle equals to electron transfer resistance $\left(R_{\mathrm{et}}\right)$. This resistance controls the electron transfer kinetic process of the redox probe at the electrode interface. The linear segment at lower frequency shows a controlled diffusion process. Figure 5 exhibits impedance spectroscopy of $5.0 \times$ $10^{-3} \mathrm{~mol} / \mathrm{L}\left[\mathrm{Fe}(\mathrm{CN})_{6}\right]^{3-/ 4-}$ at different electrodes in $0.1 \mathrm{~mol} / \mathrm{L}$ $\mathrm{KCl}$ solution. In Fig. 5, the curve of a EIS of bare GC electrode (curve a) presents very low electron transfer resistance to the redox probe in the electrolyte solution. EIS of the chitosan film modified electrode (curve b) shows a much higher interfacial electron transfer resistance $(4700 \Omega)$. This may be because the chitosan is not conductive and blocks $\left[\mathrm{Fe}(\mathrm{CN})_{6}\right]^{3-/ 4-}$ to reach the electrode surface. When $\mathrm{SnO}_{2}$ NPs (curve c) and Au NPs (curve d) are modified on the surface of GCE, lower electron transfer resistances are observed, this is ascribed to good conductivity of nanoparticles. MWCNTs are introduced to the surface of the modified electrode. EIS (curve e) is very close to a straight line. This fact can be attributed to facilitation of electron transfer by high conductivity of MWCNTs. MWCNTs in multilayer films played an important role which is similar to that of a conducting and electron transfer tunnel.

\subsection{Electrochemical behaviors of UA at the different modified electrodes}

$\mathrm{CVs}$ of UA at various modified electrodes in $\mathrm{pH}$ 7.0 PBS were shown in Fig. 6, electrochemical response of UA is very poor and the anodic peak potential is high at bare GCE. It should be found that response of UA became better at the chitosan modified electrode (curve b) than that on the bare electrode (curve a). In curve c and d, they show higher electrochemical response. This is for the reason that $\mathrm{Au}$ NPs and $\mathrm{SnO}_{2}$ NPs have higher conductivity. When only MWCNTs is introduced to the electrode surface (curve e), the peak current of UA become better at the MWCNTs modified electrode (curve e) than that at the other electrode (curve b, c, d). The result shows that MWCNTs have better electrocatalytic performance for UA than $\mathrm{SnO}_{2}$ NPs and Au NPs. When Au NPs, CHIT-SnO ${ }_{2}$ and

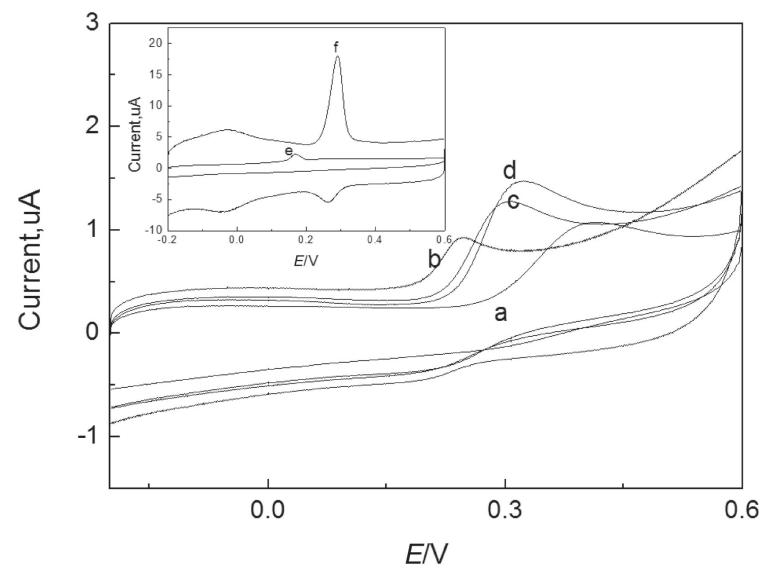

Figure 6. Cyclic voltammograms of different modified GC electrode in $2 \times 10^{-5} \mathrm{~mol} / \mathrm{L}$ UA PBS at $\mathrm{pH} 7.0$ : (a) bare GCE; (b) chitosan/GCE; (c) CHIT-SnO $/$ GCE; (d) CHIT-SnO $2 / \mathrm{Au}$ NPs/ GCE (e) MWCNTs/GCE (f) CHIT-SnO $2 / \mathrm{Au} \mathrm{NPs/CHIT-SnO}{ }_{2} /$ MWCNTs/GCE. The scan rate is $100 \mathrm{mV} / \mathrm{s}$.

MWCNTs are introduced to the electrode surface using a layer-bylayer assembled method, the peak current of UA (curve f) has been more significantly increased than that at the other modified electrode, and the peak current value is about 10 times than that at MWCNTs modified electrodes. Meanwhile, a reduction peak was observed. It should be noticed that the reduction peak of UA has rarely been observed in most literatures, but the phenomenon of uric acid reduction peak also appeared in these reports. ${ }^{37,38}$ The authors explained that the possible reason is that surface of bare electrode changes when it is modified. And modified electrode material as active center improved electron exchange reactions between the UA and the electrode, and it simultaneously improve flexibility and reversibility of the electrode reaction. It is also proposed as the main reason for the reduction of uric acid. It should be noted that the peak of the current increases sharply when $\mathrm{Au}$ NPs, CHIT-SnO $\mathrm{S}_{2}$ and MWCNTs are introduced together to the electrode surface, indicating that these different nanoparticles assembled using the layer-by-layer assembled method lead to a better electrochemical performance. Nanocomposite materials not only have advantages of $\mathrm{Au}$ NPs, CHIT-SnO 2 and MWCNTs individually, but also have multifunctional synergistic effects. The result demonstrates that the assembled composite electrode has superior electrochemical performances to those modified with MWCNTs, CHIT-SnO $\mathrm{O}_{2}$ and $\mathrm{Au}$ $\mathrm{NPs} / \mathrm{CHIT}-\mathrm{SnO}_{2}$ individually.

\subsection{Determination of UA in the presence of AA}

Uric acid (UA) is the product of catabolism of purine nucleotide and its concentration in the body should be maintained. Abnormal concentration of UA is associated with clinical situations, such as gout, hyperuricemia, Lesch-Nyan disease, cardiovascular and kidney diseases. The major problem encountered in detection of UA using electrochemical is interference from AA, which largely coexists with UA in biological fluids and has an overlapping oxidation potential on bare solid electrodes. It is important to develop a simple method to detect UA in the presence of AA. Variation of the peak current of $\mathrm{UA}$ on various layer number $\left\{\mathrm{CHIT}-\mathrm{SnO}_{2} / \mathrm{Au} \mathrm{NPs} / \mathrm{CHIT}-\mathrm{SnO}_{2}\right.$ / MWCNTs $\} n$ modified electrode with same concentration of UA indicates that the peak current at $\mathrm{n}=2$ become maximum. Therefore modified electrode with layer number of 2 is chosen for detecting UA in the next process of the experiment. Figure 7 shows DPVs of various UA concentrations in the presence of AA $(2.0 \times$ $\left.10^{-4} \mathrm{~mol} / \mathrm{L}\right)$ at $\left\{\mathrm{CHIT}-\mathrm{SnO}_{2} / \mathrm{Au}\right.$ NPs/CHIT-SnO $2 / \mathrm{MWCNTs}_{2}$. The oxidation peak potentials of AA and UA appear at -91 and $247 \mathrm{mV}$ vs. SCE on differential pulse voltammograms (DPVs), 


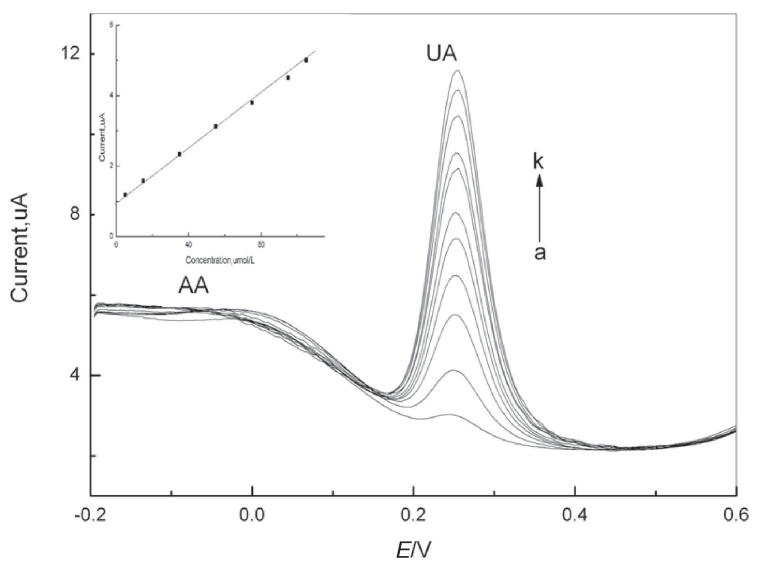

Figure 7. Differential pulse voltammograms of UA at the \{CHIT$\left.\mathrm{SnO}_{2} / \mathrm{Au} \mathrm{NPs} / \mathrm{CHIT}-\mathrm{SnO}_{2} / \mathrm{MWCNTs}\right\}_{2}$ multilayer films modified electrode in the presence of AA in PBS (pH 7.0) UA centrations: 5, $15,25,35,45,55,65,75,85,95,105 \mu \mathrm{mol} / \mathrm{L}$.

respectively. The result indicates that there is no interference on determination of UA in the presence of AA. The response of UA is linear with its concentrations in the range from $4.0 \times 10^{-6}$ to $3.0 \times$ $10^{-3} \mathrm{~mol} / \mathrm{L}$ and the linear regression equation is $\mathrm{i}_{\mathrm{pa}}(\mu \mathrm{A})=1.027+$ $0.037 \mathrm{C}(\mu \mathrm{mol} / \mathrm{L})$, with a correlation coefficient of 0.992 . The detection limit for $\mathrm{UA}$ is $2.0 \times 10^{-6} \mathrm{~mol} / \mathrm{L}(\mathrm{S} / \mathrm{N}=3)$.

\subsection{Stability of the $\left\{\mathrm{CHIT}-\mathrm{SnO}_{2} / \mathrm{Au} \mathrm{NPs} / \mathrm{CHIT}-\mathrm{SnO}_{2} /\right.$ MWCNTs $\}_{2}$ modified electrodes}

Stability of a modified electrode is an essential issue for practical applications. However, the LB multilayer, casting film and even some self-assembled monolayer modified electrodes show poor stability in electrolyte solution. Here, we compare the stability of the casting film on multilayer films modified electrodes by CV. After scanning 300 circles for $\mathrm{CVs}$ at modified electrodes, the peak current of UA $\left(2.0 \times 10^{-5} \mathrm{~mol} / \mathrm{L}\right)$ decreases to $96 \%$ of that at fresh modified electrodes. This tiny change indicates that the modified electrodes can not only act as electron mediator, but also have good stability and sensitivity. They can be used as multilayer modified electrodes to analysis UA.

\section{Conclusion}

We assembled thin, uniform and stable $\{$ CHIT-SnO $2 / \mathrm{Au}$ NPs/ CHIT-SnO $2 / \mathrm{MWCNTs}_{\mathrm{n}}$ multilayer films. Same amounts of MWCNTs and gold nanoparticles were assembled during each cycle. $\left\{\mathrm{CHIT}-\mathrm{SnO}_{2} / \mathrm{Au} \mathrm{NPs} / \mathrm{CHIT}-\mathrm{SnO}_{2} / \mathrm{MWCNTs}_{2}\right.$ film modified $\mathrm{GC}$ was used as a working electrode, and it exhibited a remarkably good selectivity towards UA detection. The result of differential pulse voltammetry showed that AA has no interference with UA detection.

\section{Acknowledgments}

This work was supported by National Natural Science Foundation of China (11204005), the Provincial Natural Science Research
Program of Higher Education Institutions of Anhui province (KJ2012Z034) and Anhui Provincial Natural Science Foundation (1308085QA08).

\section{References}

1. C. N. R. Rao, B. Satishkumar, A. Govindaraj, and M. Nath, ChemPhysChem, 2, 78 (2001).

2. R. H. Baughman, A. A. Zakhidov, and W. A. de Heer, Science, 297, 787 (2002).

3. M. Musameh, J. Wang, A. Merkoci, and Y. Lin, Electrochem. Commun., 4, 743 (2002).

4. F.-H. Wu, G.-C. Zhao, and X.-W. Wei, Electrochem. Commun., 4, 690 (2002).

5. K. Wu, Y. Sun, and S. Hu, Sens. Actuators, B, 96, 658 (2003).

6. J. Wang, M. Li, Z. Shi, N. Li, and Z. Gu, Electrochim. Acta, 47, 651 (2001).

7. M. A. Correa-Duarte, A. Kosiorek, W. Kandulski, M. Giersig, and L. M. LizMarzan, Chem. Mater., 17, 3268 (2005).

8. J. Wang, M. Musameh, and Y. Lin, J. Am. Chem. Soc., 125, 2408 (2003).

9. J. H. Rouse and P. T. Lillehei, Nano Lett., 3, 59 (2003).

10. H. Paloniemi, M. Lukkarinen, T. Ääritalo, S. Areva, J. Leiro, M. Heinonen, K. Haapakka, and J. Lukkari, Langmuir, 22, 74 (2006).

11. J. Shi, E. S. McLamore, D. Jaroch, J. C. Claussen, R. G. Mirmira, J. L. Rickus, and D. M. Porterfield, Anal. Biochem., 411, 185 (2011).

12. T. Zhang, W. Wang, D. Zhang, X. Zhang, Y. Ma, Y. Zhou, and L. Qi, Adv. Funct. Mater, 20, 1152 (2010)

13. M. Acciarri, C. Canevali, C. Mari, M. Mattoni, R. Ruffo, R. Scotti, F. Morazzoni, D. Barreca, L. Armelao, and E. Tondello, Chem. Mater, 15, 2646 (2003).

14. K. Yu, Y. Xiong, Y. Liu, and C. Xiong, Phys. Rev. B, 55, 2666 (1997).

15. E. Topoglidis, Y. Astuti, F. Duriaux, M. Grätzel, and J. R. Durrant, Langmuir, 19, 6894 (2003).

16. A. Grenha, C. I. Grainger, L. A. Dailey, B. Seijo, G. P. Martin, C. RemuñánLópez, and B. Forbes, Eur. J. Pharm. Sci., 31, 73 (2007).

17. D.-H. Li, L.-M. Liu, K.-L. Tian, J.-C. Liu, and X.-Q. Fan, Carbohydr. Polym., 67, 40 (2007).

18. J. Hodak, R. Etchenique, E. J. Calvo, K. Singhal, and P. N. Bartlett, Langmuir, 13, 2708 (1997).

19. Y. Sun, J. Sun, X. Zhang, C. Sun, Y. Wang, and J. Shen, Thin Solid Films, 327329, 730 (1998).

20. E. Calvo, R. Etchenique, L. Pietrasanta, A. Wolosiuk, and C. Danilowicz, Anal. Chem., 73, 1161 (2001).

21. Y. Lvov, G. Decher, and H. Moehwald, Langmuir, 9, 481 (1993)

22. G. Decher, Science, 277, 1232 (1997).

23. B.-Y. Wu, S.-H. Hou, F. Yin, Z.-X. Zhao, Y.-Y. Wang, X.-S. Wang, and Q. Chen, Biosens. Bioelectron., 22, 2854 (2007).

24. L. Wang, S. Guo, L. Huang, and S. Dong, Electrochem. Commun., 9, 827 (2007).

25. J. J. Storhoff, R. Elghanian, R. C. Mucic, C. A. Mirkin, and R. L. Letsinger, J. Am. Chem. Soc., 120, 1959 (1998).

26. R. C. Engstrom and V. A. Strasser, Anal. Chem., 56, 136 (1984).

27. K. Sundberg, W. Smyrl, L. Atanasoska, and R. Atanasoski, J. Electrochem. Soc., 136, 434 (1989).

28. L. J. Kepley and A. J. Bard, Anal. Chem., 60, 1459 (1988).

29. G. Di Carlo, A. Curulli, R. G. Toro, C. Bianchini, T. De Caro, G. Padeletti, D. Zane, and G. M. Ingo, Langmuir, 28, 5471 (2012).

30. A. Curulli, F. Valentini, G. Padeletti, A. Cusma, G. Ingo, S. Kaciulis, D. Caschera, and G. Palleschi, Sens. Actuators, B, 111-112, 526 (2005).

31. M. Cui, J. Huang, Y. Wang, Y. Wu, and X. Luo, Biosens. Bioelectron., 68, 563 (2015).

32. Y. Liu, Y. Liu, H. Feng, Y. Wu, L. Joshi, X. Zeng, and J. Li, Biosens. Bioelectron., 35, 63 (2012).

33. M. Mallesha, R. Manjunatha, C. Nethravathi, G. S. Suresh, M. Rajamathi, J. S. Melo, and T. V. Venkatesha, Bioelectrochem., 81, 104 (2011).

34. S. Liu, J. Yan, G. He, D. Zhong, J. Chen, L. Shi, X. Zhou, and H. Jiang, J. Electroanal. Chem., 672, 40 (2012).

35. G. Zeng, Y. Xing, J. Gao, Z. Wang, and X. Zhang, Langmuir, 26, 15022 (2010).

36. E. A. Hall, N. G. Skinner, C. Jung, and S. Szunerits, Electroanalysis, 7, 830 (1995).

37. S. Qi, B. Zhao, H. Tang, and X. Jiang, Electrochim. Acta, 161, 395 (2015).

38. C. Wang, Master thesis, University of Science and Techonoly of China (2006). 